\title{
Una mirada a la comunicación para el cambio social como generadora de participación y empoderamiento político de la población infantil de Cartagena a través de la implementación de estrategias del eduentretenimiento
}

\section{A look at the communication for social change as a generator of participation and political empowerment of the child population in Cartagena through the implementation of strategies of edutainment.}

rnvarrod@hotmail.com, Doctorando en Ciencias Sociales de la Universidad del Norte. Magister en comunicación de la Universidad del Norte. \author{
Profesor de la Universidad Sergio Arboleda de Santa Marta \\ Luis Ricardo Navarro Díaz \\ anyo_c@hotmail.com, Comunicadora Social de la Universidad Tecnológica de Bolivar \\ Angélica Castellar Cuervo \\ belleza-amarga@hotmail.com, Comunicadora Social de la Universidad Tecnológica de Bolívar
}

Swanny Liv Rocha Acosta

Recibido Abril 4 de 2012

Aceptado Mayo10 de 2012

\begin{abstract}
RESUMEN
El artículo recoge el análisis e implementación de estrategias de eduentretenimiento como dinámicas de comunicación pertinentes para generar procesos de participación y empoderamiento político en niños del sector de La Boquilla de Cartagena de Indias. La investigación asume la necesidad de desarrollar procesos mediados por la comunicación, transformadores de las relaciones políticas entre los actores sociales, en este caso con niños. El estudio, de diseño cualitativo, fue realizado durante el año 2011 con el apoyo de la Universidad Tecnológica de Bolívar y su programa de Comunicación Social. Su paradigma interpretativo, ya que el investigador hace su propia descripción y valoración de los datos La técnica empleada fue la observación y se emplearon talleres lúdicos. Se resalta como principal conclusión la importancia de la comunicación y el eduentretenimiento, en el proceso de reconocimiento de la diferencia, y en la posibilidad de asumir relaciones sociales que fortalezcan el tejido social específico de La Boquilla de Cartagena-Bolívar
\end{abstract}

Palabras clave: eduentretenimiento, comunicación para el cambio social, participación y empoderamiento.

\begin{abstract}
This article includes the analysis and implementation of strategies of edutainment as relevant communication Dynamics to generate processes of participation and political empowerment in the nozzle of Cartagena de Indias sector children. The research assumes the necessity of developing processes mediated by communication, transformers of the political relations between the social actors, in this case with children. The study, qualitative design, was made during the year 2011 with the support of the technological University of Bolivar and his program of Social communication. His paradigm interpretation, since the researcher makes its own description and assessment of the technical data: observation and playful workshops. As main conclusion highlights the importance of communication and edutainment, in the process of recognition of the difference, and the possibility of assuming social relationships that strengthen the specific social fabric of the nozzle of Cartagena - Bolivar
\end{abstract}

Key words: edutainment, communication for social change, participation and empowerment 


\section{Introducción}

Como punto de partida es relevante delimitar el área de trabajo del siguiente documento; para ello es pertinente exponer que el estudio se encuentra adscrito al área de la comunicación para el cambio social $^{1}$, y específicamente a la temática relacionada con lo que algunos teóricos asocian como "Comunicación y educación" (Kaplún, 2002, p. 13). Por su parte, Alfonso Gumucio en su texto Haciendo Olas (2001b) lo comprueba presentado 50 experiencias de comunicación para el desarrollo y cambio social en diversas partes del mundo, con diversos formatos y maneras de comunicar. En coherencia con esto, Restrepo define la comunicación para el desarrollo como "el uso de los procesos, técnicas y medios de comunicación para ayudar a las personas a tomar plena conciencia de su situación y sus opciones de cambio, para resolver conflictos, para trabajar hacia un consenso, para ayudarlas a planificar acciones de cambio y desarrollo sostenible, a adquirir los conocimientos y habilidades que necesitan para mejorar su condición y la de la sociedad, y para mejorar la eficacia de las instituciones" (1998, p. 793). En este caso, la experiencia que aquí se describe realiza un seguimiento a dos categorías concretas a saber: participación y empoderamiento en niños de una escuela pública del sector de la Boquilla de la ciudad de Cartagena de Indias durante el año 2011. La propuesta apunta a entender que los procesos de participación de una comunidad tienen íntima relación con los procesos de cambio social, lo que implica un sujeto también con ciertas condiciones. De allí la pertinencia del trabajo con niños. El cambio fundamental aquí consiste en el paso de un sujeto acrítico a un sujeto crítico (Navarro, 2008, p. 338); de un sujeto a quien los condicionamientos de su medio lo han hecho pasivo, conformista, fatalista, a un sujeto que asuma su propio destino; un sujeto capaz de superar sus tendencias egoístas e individualistas y abrirse a los valores solidarios y comunitarios. Esto sugiere la necesidad de volver a la enseñanza de las Ciencias Sociales a partir de la teoría social crítica, lo que supondría en palabras de Kemmis (1996):

...la crítica ideológica a la naturaleza, a las relaciones sociales de producción, reproducción y transformación social, y la organización de la acción social y política para mejorar el mundo, a partir de la idea dialéctica de racionalidad y por la idea comunitaria e igualitaria de justicia y libertad. (p. 31, citado en Ardila, 2010, p. 50).

Esto se puede apoyar en la siguiente cita del argentino Mario Kaplún (1923-1998) cuando aseguró que: “...aún para alcanzar las metas de un desarrollo puramente cuantitativo y material, se necesita una honda transformación en la educación del pueblo, que haga hombres pensantes, capaces de participar activamente en el proceso, más responsables y más creativos" (1978a, p. 251). Para lograrlo, es necesario integrar procesos particulares de educación. Este académico uruguayo diseñó a finales de la década

Aunque en este artículo se ofrecen definiciones y aproximaciones teóricas a la comunicación para el cambio social, para profundizar sobre sus alcances, métodos y objetivos sugiero el texto Antología de la comunicación para el cambio social editado en 2008 por Alfonso Gumucio y Tomas Tufte . de los setenta la estrategia llamada Cassete foro rural (1978b), procedimiento simple y barato que facilitó el diálogo a distancia entre cooperativas de agricultores.

El mismo Kaplún (2002, p. 20) destacaba la necesidad de una educación transformadora de las personas y las comunidades; una educación preocupada no tanto por los contenidos transmitidos, ni por el comportamiento o reacción generada, sino más bien por las interacciones logradas entre los individuos y su realidad. Por su parte, Kaplún influenciado por las propuestas de Paulo Freire (2004) y Juan Díaz Bordenave (1976) propone distinguir tres tipos de educación, entre los cuales debemos optar. Por una parte la que pone el énfasis en los contenidos; por otro, la que pone el énfasis en los resultados; y por último, la que pone el énfasis en el proceso (Kaplún, 1978a, p. 246).

Ahora bien, Paulo Freire propone la comunicación como un elemento central de su planteamiento sobre educación. "Como señaló Jarbas Maciel, quien fuera colaboradora del maestro de Pernambuco: "La categoría fundamental en la que está inmerso el Sistema de Educación Paulo Freire es la categoría sociológica y antropocultural de la comunicación". Al describir el concepto antropológico de cultura tal como lo esboza Freire, Maciel sostiene que el tema central es esa idea inicial: el hombre es un ser de relaciones" (Marques de Melo, 1979, p. 279). Desde aquí es posible inferir que para Freire, la cultura del silencio, que impone y reproduce la opresión, es una característica de todos los pueblos colonizados y tiene sus raíces en la práctica secular de la incomunicación. Desde el punto de vista del presente estudio, la Boquilla no es la excepción. No es difícil establecer una conclusión inmediata: los colonizados son según Freire, silentes, carecen de voz propia, hablan por medio del discurso del opresor. De esta forma lo sustenta el autor en su texto $L a$ educación como práctica de la libertad: "Las sociedades a las que se les niega el diálogo, la comunicación, y en su lugar se les ofrece comunicados, resultantes de la compulsión o la donación deviene preponderantemente que carece de un contenido marcadamente crítico" (Freire, 2007, p. 63).

$\mathrm{Al}$ respecto, el brasileño José Marques de Melo (1943- ) en un texto titulado La comunicación en la pedagogía de Pablo Freire (1979), aseguraba que Freire vuelve más explícita la naturaleza de su pedagogía de la comunicación al implicar una comunión entre educador y educando, lo que exige una pedagogía abierta, en constante reformulación. Esa fue la pretensión del trabajo de campo realizado en el sector de la Boquilla. Cuanto más intensa la comunicación, más rica la interacción de los participantes. En relación con este planteamiento, una de las tesis más importantes de sus documentos apunta a asociar la comunicación con la libertad. "La comunicación y la libertad son fenómenos concomitantes y no dicotómicos. La comunicación se produce automáticamente sólo con libertad. A su vez, la libertad se conquista únicamente con la comunicación" (Marques de Melo, 1979, p. 283). Por su parte, Freire (1970) en su texto Pedagogía del oprimido, también lo expone de la siguiente forma: "La libertad se consigue con- 
quistándola, no por un regalo. Debe buscarse constantemente (...) Es más bien, la condición indispensable para la búsqueda en que estamos inscritos los hombres como seres inconclusos" (1979, p. 32). A partir de estas reflexiones teóricas, el estudio analiza procesos de participación y empoderamiento en las dinámicas de educación de niños y niñas, del sector de La Boquilla de Cartagena, con edades comprendidas entre 6 y 13 años, con el fin de generar a partir de propuestas comunicativas dinámicas de participación y empoderamiento político en esos contextos sociales, particularmente en actores sociales de la Institución Técnica de la Boquilla- INETEB.

\section{Metodología}

Se propuso, entonces, una metodología distinta para establecer soluciones pedagógicas de tipo no formal a problemas de la vida cotidiana de estos actores sociales, asumiendo en este caso el eduentretenimiento como vehículo facilitador de este proceso. Dado lo anterior, la investigación giró entorno al siguiente interrogante: ¿cuáles son las estrategias de eduentretenimiento pertinentes para transformar las dinámicas comunicativas de participación y empoderamiento en la educación de niños y niñas entre 6 y 13 años del sector La Boquilla de Cartagena? En este sentido, se hace necesario contextualizar el lugar en donde se desarrolló la investigación. Para ello, el primer segmento de este documento expone un recuento sobre este particular; en él se visualizan aspectos relacionados con la historia, la geografía, la educación y la sociología de las comunidades en donde se realizó el trabajo. De la misma forma, en un segundo apartado, el artículo presenta el sustento teórico sobre el cual se construye el trabajo de campo. En este sentido, se hace referencia a pensadores como Mario Kaplún (2002), Gita Sen (1995), Thomas Tuffte (2005) y Jesús Martín Barbero (1987), de cuyas propuestas fueron extraídas las categorías centrales para el estudio, y entre las cuales es posible citar educación, participación y empoderamiento político.

En este orden de ideas, el presente documento tiene como objetivo describir reflexivamente el análisis e implementación de estrategias de eduentretenimiento como dinámicas de comunicación pertinentes para generar procesos de participación y empoderamiento político en niños del sector de La Boquilla de Cartagena de Indias. A su vez, la búsqueda de nuevas formas de lograr objetivos educativos, ya no desde lo tradicional, unidireccional y transmisionista, obligó al planteamiento de actividades propuestas desde tres ejes centrales a saber: participación, reconocimiento del otro y democracia. La primera tarea consistió en que los menores aprendieran los significados de estos conceptos y así pudieran narrar, desde estilos y entornos propios, dichos elementos.

Según lo anterior, la categoría "educación no formal" se ofrece como un concepto central de la investigación, por lo que se hace necesario delimitarla y exponer su apuesta de manera inmediata. Por educación no formal el estudio asume aquella "actividad que se ofrece con el objeto de complementar, actualizar, suplir conocimientos y formar en aspectos académicos o laborales sin estar sujetas al sistema de niveles y grados establecidos para la educación formal" (Ministerio de Educación Nacional, Parra. 4, Ley 115 de 1994). Dado lo anterior, es posible asumir como forma de educación no formal las actividades propuestas desde la base teórica del eduentretenimiento. De hecho los medios masivos intentan implementarlo. Relacionada con esta perspectiva se encuentra otra categoría clave en la propuesta del estudio como es la participación. El académico colombiano Manuel Vidal en la Revista Signo y Pensamiento (1991), propone tres tipos de participación: 1. Participar es notifica, avisar, hacer saber, 2. Participar es cooperar, contribuir, hacer parte y 3. Participar es comunicar, intervenir y tomar parte. Esta última es aquella en la cual hay comunicación e intervención de todos en la solución de un conflicto, en todas las etapas del mismo desde su definición hasta su solución, y por tanto, fue la implementada en el desarrollo de la investigación.

Por último, y como asociada a los procesos de eduentretenimiento, es válido mencionar la otra categoría central del estudio como fue la de empoderamiento político la cual se relaciona antes que nada con el poder, en la medida en que supone un cambio de las relaciones de poder en favor de aquellos que con anterioridad tenían escasa autoridad sobre sus propias vidas. "Del empoderamiento se pueden extraer poderosas reservas de esperanza y entusiasmo entre las personas que estaban acostumbradas a verse a sí mismas y a sus mundos en términos puramente negativos. Los agentes de cambio externo pueden requerirse como los catalizadores esenciales que lo inician, pero el impulso del proceso de empoderamiento se explica por la extensión o la rapidez con que las personas se cambian a sí mismas" (Sen, 1995, p. 2).

\section{Talleres Lúdicos}

Una vez definidas y fundamentadas cada una de las anteriores categorías se dio lugar al diseño, realización y ejecución de cinco talleres los cuales fueron aplicados a niños y niñas entre 6 y 13 años del sector de La Boquilla de Cartagena de Indias, abordando lúdicamente las temáticas de los tres vectores centrales de la investigación propuestos desde el inicio de la misma: participación, reconocimiento del otro y democracia. Ahora bien, para el desarrollo de este trabajo, el eduentretenimiento se asume como el uso del entretenimiento como una práctica comunicacional específica generada para comunicar estratégicamente respecto de cuestiones del desarrollo; se trata de la posibilidad de articular agendas para el cambio social con la vida cotidiana de la gente a través de la sumatoria de dos elementos fundamentales: educación y entretenimiento. De esta forma se configuran también esfera públicas y se generan ciudadanos transformadores y participativos dentro de los tejidos sociales (Navarro, 2010b, p. 37). En ese sentido, el diseño de cinco talleres a partir de esta concepción de eduentretenimiento dio lugar a la construcción de diversas actividades lúdicas de comunicación que incluyeron rallys, conversatorios, dinámicas en grupo e individuales, propuestas de comunicación verbal (poemas, canciones, etc.) y de 
comunicación no verbal (dramatizados, bailes, dibujos). Para finalizar, y a partir de este recorrido, el documento ofrece en su último segmento los resultados y recomendaciones descriptivas para implementar estrategias de eduentretenimiento en La Boquilla, como forma de transformar la educación tradicional en educación para el desarrollo.

Por su parte, y antes de exponer el planteamiento del problema trabajado, cabe resaltar que el profesor danés Thomas Tuffte ${ }^{2}$ ha sido pionero en este tipo de investigación. Con su publicación El eduentretenimiento en la comunicación para el VIH/sida, ha sustentado la combinación del entretenimiento con la educación de manera integral, la mayoría de las veces utilizando dramas radiales y televisivos, pero también géneros musicales, teatro y talk shows. El resultado es un número creciente de productos de medios diseñados con el objetivo específico de educar a las audiencias. Por su parte, en Latinoamérica los estudios relacionados al eduentretenimiento han sido notorios, en especial en temas relacionados con la salud, en tópicos que van desde presión arterial, consumo de tabaco y promoción de vacunas, hasta la planificación familiar y la prevención del VIH/Sida. En el plano particular de Cartagena es posible asegurar que aún no existen investigaciones académicas relacionadas con la temática del eduentretenimiento y la comunicación, lo que hace pertinente el desarrollo e implementación de estrategias de comunicación para generar dinámicas alternativas de educación basadas en la participación y empoderamiento político en niños del sector de La Boquilla de Cartagena de Indias, tal como lo plantea el siguiente desarrollo. Esta propuesta se construye desde la orilla opuesta a la comunicación de masas. "Seguir llamando medios de comunicación a todos los medios de masas no facilita la creación de auténticos medios para la construcción social y la participación activa, lúdica y crítica de la ciudadanía" (Chaparro, 2009, p. 148). Se hace necesario pensar en otras dinámicas de comunicación, en otros medios de comunicación para crear sujetos con propuestas alternativas, ciudadanos políticos y por ende participativos.

\section{Aspectos teóricos del estudio}

El estudio propone articular la categoría de eduentretenimiento sustentada a partir de la propuesta de Thomas Tuffte, y citada en el segmento anterior, con los conceptos de empoderamiento y participación a partir de su dimensión comunicativa. Para este

\footnotetext{
Tiene una maestría en sociología cultural (1989) y un doctorado en comunicación (1995), comenzó su trabajo como profesor en el RUC en la comunicación en mayo de 2004. Sus áreas de investigación incluyen la comunicación para el desarrollo y el cambio social, la comunicación para la salud, la comunicación participativa, los estudios cualitativos de audiencia (recepción y análisis de la etnografía de audiencia), los medios de comunicación, la sociología, la globalización cultural y el origen étnico. Sus Principales proyectos de investigación se han ocupado de: telenovelas, la cultura y el desarrollo en América Latina (1989-1994), utiliza los medios de comunicación, nuevas tecnologías y la identidad cultural en Brasil (1996-1999), esgrime los medios de comunicación, la vida cotidiana y la formación de la identidad entre los jóvenes de las minorías étnicas en Copenhague (1999-2001) y el VIH/SIDA en Sudáfrica Comunicación- una crítica del paradigma dominante (2001-2005).
}

efecto, es necesario sustentar estas dos categorías. Como punto de partida es válido tomar la propuesta que Kaplún realiza en su libro Hacia nuevas estrategias de comunicación en la educación para adultos, en donde hace énfasis crítico contra la educación masiva instruccional. En relación con ello, el estudio propone comprender la comunicación como un proceso de transformación de las personas y de las sociedades. Tal como se propone en este artículo, lo crítico se puede interpretar, en ámbitos educativos a través de "la importancia de identificar problemas, cuestionarse, cuestionar la realidad social y plantear alternativas de solución de manera reflexiva, crítica e indagadora, y proponer la reflexión constante para analizar procesos y generar verdaderos cambios" (Moreno, 2002, p. 42).

Esto implica que el alumno o receptor piense por sí mismo con el fin de generar acciones que transformen su realidad (Kaplún 1983, p. 24). Así mismo, según Martín Barbero (2002b) en su libro La educación desde la comunicación, asegura que la manera como la escuela se empeña en seguir con la educación tradicional, implica abordar las temáticas a partir de la cultura del silencio, es decir, desde el conjunto de acciones y esquemas de pensamientos, mentalidades y comportamientos de la sociedad latinoamericana oprimida desde la conquista.

De esta forma, el eduentretenimiento se asume como estrategia de enseñanza, que plantea procesos de educación generadores de dinámicas de empoderamiento en los receptores, que para el caso particular del estudio son los niños del sector de La Boquilla. Ahora bien, Gita Sen propone el empoderamiento como una categoría relacionada con el poder, es decir, el empoderamiento se relaciona, primero y antes que nada, con el poder, cambiando las relaciones de poder en favor de aquellos que con anterioridad tenían escasa autoridad sobre sus propias vidas. Batliwala (1993,) lo define como poseedor de dos aspectos centrales: control sobre los recursos (físicos, humanos, intelectuales, financieros, y el de su propio ser), y control sobre la ideología (creencias, valores y actitudes). "Si el poder significa control, el empoderamiento, por tanto, es el proceso de ganar control" (Sen, 1995, p. 2).

Lo anterior aplicado al estudio, buscó facilitar procesos de transformación de niños y niñas entre 6 y 13 años del sector La Boquilla de Cartagena. En estas poblaciones, las problemáticas que se hacen más evidentes entre los niños y niñas participantes del estudio, son las relacionadas a los problemas de sociabilidad, costumbres violentas y poca participación en los procesos en favor de la comunidad. Para tales problemas, la participación y el empoderamiento político a través de la implementación del eduentretenimiento se proponen como conceptos desde los cuales es posible repensar el tejido social, de tal manera que sea posible proponer procesos que conlleven a toma de decisiones propias y creatividad para enfrentar los problemas sociales. Tal como lo asegura Gita Sen:

Del empoderamiento se pueden extraer poderosas reservas de esperanza y entusiasmo entre las personas que estaban acostum- 
bradas a verse a sí mismas y a sus mundos en términos puramente negativos. Los agentes de cambio externo pueden requerirse como los catalizadores esenciales que lo inician, pero el impulso del proceso de empoderamiento se explica por la extensión o la rapidez con que las personas se cambian a sí mismas (1995, p. 2).

En resumen, el empoderamiento se relaciona con la participación y aunque a menudo estos conceptos son usados como sinónimos, tienen significados distintos, puesto que el empoderamiento (de aquellos que previamente carecían de poder en el orden social) es un fin en sí mismo, mientras que la descentralización y la participación, pueden ser utilizadas como medios para un fin. En este contexto teórico es pertinente ubicar el espacio en donde se realizó el estudio. El problema de este estudio se establece en la zona de La Boquilla, la cual es una comunidad de pescadores, del norte de la ciudad de Cartagena de Indias. Esta población data de 200 años aproximadamente. La Boquilla recibe este nombre debido a su posición geográfica, ya que ésta se encuentra sobre un banco de arena que separó del mar la laguna costera llamada Ciénaga de La Virgen comunicada con el Mar Caribe por una serie de canales llamados bocas. De allí el nombre de La Boquilla, por ser la boca más pequeña de las restantes Boca grande y Boca chica. Esta población cuenta con la Institución Educativa Técnica de la Boquilla (INETEB), la cual ofrece a la comunidad y sus zonas aledañas educación preescolar, básica primaria y secundaria, media técnica y académica dirigida a niños, jóvenes y adultos. Así pues, esta institución cuenta con dos aulas por cada grado. El estudio seleccionó dos salones entre segundo y tercer gado, constituyendo una muestra de 25 niños y niñas de segundo grado de primaria con edades entre 6 y 8 años del INETEB. La segunda muestra fue conformada por 28 niños y niñas de tercer grado de primaria de 8 a 12 años de la misma Institución, pertenecientes a los estratos 0 y 1 (bajo), pero identificados por sus profesores con altos índices de indisciplina.

\section{Una mirada a La Boquilla}

La Boquilla es una comunidad de pescadores, ubicada al norte de la ciudad de Cartagena de Indias. Este corregimiento en épocas de la Colonia fue habitado por la tribu Caribe que tuviera allí sus asentamientos. En la actualidad se han encontrado vestigios arqueológicos, que datan de la Cartagena fortificada. Los Corsarios y piratas, entraban en pequeñas embarcaciones por La Boquilla, atravesaban la Ciénaga y sitiaban a la ciudad por el nororiente, por lo que se hizo necesario construir baterías en la entrada de las bocas, que hoy yacen sepultadas bajo tierra. Posteriormente esta área fue poblada por algunas familias provenientes de pueblos como San Onofre (sucre), Villanueva (Bolívar) y Rocha. Estos pobladores se establecieron en ranchos de bahareque, y su fuente productiva fue el desarrollo de la pesca.

Dentro de su organización social la familia es una variable importante a la hora de estudiar una comunidad, porque ésta es la institución más importante y básica de la sociedad. Por esto se ha considerado como un garante del desarrollo integral del indi- viduo, y por lo tanto su estructura, funcionamiento y ciclo vital han sido modificados y adaptados de acuerdo con las condiciones sociales, económicas, políticas y culturales. En la actualidad, las familias boquilleras, son amenazadas por el fenómeno de descomposición, arrastradas por los problemas del mundo contemporáneo embarazos a temprana edad, consumo de sustancias psicoactivas, alcoholismo, muchos de ellos en menores de edad. La comunidad no posee alternativas de recreación, esparcimiento y actividades productivas.

\section{La Boquilla Afro descendiente}

La Boquilla es Afrocaribe y afrocolombiana. Una muestra de ello es que el $99 \%$ de los 2742 estudiantes de la principal institución educativa (La INETEB) pertenezcan a este grupo. En esta comunidad se conjugan el mar, la ciénaga de la virgen, el sol, el sabor caribe y la fuerza de los ritmos africanos. La Boquilla, al ser una comunidad afrodescendiente, ha permitido que su población, pese a los escasos recursos y oportunidades, se mantenga firme, con brazos abiertos y sonrisa amable. Que sus niños, antes de aprender a caminar, aprendan a moverse al son de una champeta o de un ritmo alegre. Que convivan en armonía con personas de distintas regiones y ciudades de Colombia. En el campo educativo La Boquilla cuenta con un comité de etnoeducación, cuyo principal objetivo es, desde la academia, propender por la preservación de sus raíces, costumbres, tradición oral, entre otras que configuran su identidad.

\section{Educación en la Boquilla}

Es pertinente la postura socio -humanista en la cual "La educación debe permitirles tomar conciencia de sí mismos y de sus posibilidades desde la acción de su propio medio. Trata de enseñar a los hombres liberarse de la "colonización del espíritu", a través de la proposición de un concepto humanista y liberador de la educación" (Freire, 2003). Desde esta cita, es posible también contextualizar algunas características de la institución educativa en donde se llevó a cabo el estudio, como las siguientes:

Visión: la Institución Educativa Técnica de la Boquilla es en el 2010 un centro de excelente calidad educativa que goza de amplio reconocimiento a nivel local y nacional por su formación en su educación media técnica y académica y, el desarrollo socio humanístico orientado a mejorar el nivel de vida del estudiante y su comunidad.

Misión: en la Institución Educativa Técnica de la Boquilla se ofrece a la comunidad de la Boquilla y sus zonas aledañas, educación pre-escolar, básica primaria y secundaria, media técnica y académica dirigida a niños, jóvenes y adultos para lograr mediante modelos de gestión participativa y democrática, una formación integral que les facilite contribuir a la transformación cultural y social de su entorno y, a la adecuada atención de sus necesidades y problemas mejorando así su calidad de vida. 
El modelo pedagógico de la institución está orientado desde esta visión y misión el cual se implementa en un contexto desfavorecido económicamente pero rico culturalmente. Es importante destacar el compromiso con el desarrollo del mismo a través de la aplicación de un modelo pedagógico "cognitivo socio- humanístico con carácter participativo y democrático" que permitirá la formación de jóvenes integrales capaces de lograr mediante modelos de gestión participativa democrática una formación tal, que posibilite la transformación tanto de sí mismo como de su entorno.

\section{El eduentretenimiento y su relación con los conceptos de comunicación, participación y empoderamiento político.}

En este segmento del documento se establecen los postulados teóricos que ayudaron a sustentar la investigación, así como los autores que dieron soporte a las categorías implementadas. A partir de esto, se utilizó el concepto de manera articulada al discurso de la comunicación para el cambio social, según lo propuesto por Thomas Tufte (2005, p. 16), y definido como "el uso del entretenimiento como una práctica comunicacional específica generada para comunicar estratégicamente, respecto de cuestiones del desarrollo en una forma y con un propósito que pueden ir desde el marketing social de comportamientos individuales en su definición más limitada, hasta la articulación de agendas en pos del cambio social liderada por los ciudadanos y con un propósito liberador". Es por eso que la comunicación que propone este trabajo concibe al ciudadano como un sujeto definido como multidimensional. "Por lo tanto, las identidades de estos sujetos no son permanentes, son más bien, fuertemente indeterminadas, cambiantes y diferenciadas, y casi siempre conflictivas" (Navarro, 2010a, p. 185).

Esta concepción de comunicación se articuló con los conceptos de empoderamiento político y participación, siempre teniendo en cuenta su dimensión comunicativa; en este contexto, Gita Sen propone el empoderamiento como una herramienta de poder, es decir, "el empoderamiento se relaciona, primero y antes que nada, con el poder, cambiando las relaciones de poder en favor de aquellos que con anterioridad tenían escasa autoridad sobre sus propias vidas. De igual forma Batliwala (1993, p. 1) lo define como poseedor de dos aspectos centrales: control sobre los recursos (físicos, humanos, intelectuales, financieros, y el de su propio ser), y control sobre la ideología (creencias, valores y actitudes). Si el poder significa control, el empoderamiento, por tanto, es el proceso de ganar control".

Por consiguiente, y de acuerdo con lo anterior, se hace evidente en esta investigación de carácter cualitativa, la contraposición al patrón vertical y tradicional de educación que utiliza la institución INETEB, que según Kaplún en su libro Hacia nuevas estrategias de comunicación en la educación de adultos define como pedagogía trasmisora (énfasis en los contenidos) y que corresponde a la educación tradicional basada esencialmente en la transmisión de conocimientos y valores de una generación a otra, del profesor al alumno, de la élite a la masa. Su objetivo es, pues, que el alumno “aprenda". Desde esta tesis se proponen las siguientes categorías como centrales en el desarrollo del estudio:

\section{El eduentretenimiento como vehículo facilitador de cambio social}

El eduentretenimiento es concebido, dentro de la investigación como el uso del entretenimiento como una práctica comunicacional específica generada para comunicar estratégicamente respecto de cuestiones del desarrollo. (Tufte, 2008). A partir de esta noción, se hace posible afirmar que el eduentretenimiento es buscar que por medio de los mensajes comunicacionales se logre educar a la audiencia, con el fin de crear actitudes favorables y así cambiar un comportamiento establecido. Desde Martín Barbero, la tarea de estudios como el que se presenta en este documento se puede resumir mediante la siguiente cita:

...necesitamos pensar las industrias culturales aliviadas del "peso de la gravedad causal", esto de "su remisión en cadena a las totalidades"3, para concebirlas más bien como lugares de condensación e interacción de redes culturales múltiples, de entrecruzamiento de diferentes espacios de la producción social, conformadas por dispositivos complejos que no son de orden meramente tecnológico, mercantil o político, y en las que pesan menos las filiaciones que las alianzas, las pesadas máquinas de la fabricación que las sinuosas trayectorias de la circulación y donde las estratagemas de la apropiación deben ser tenidas en cuenta tanto como las lógicas de la propiedad (Barbero, 1999, p. 2)

En este orden de ideas, es fundamental citar un verdadero hito de la literatura escrito por Jesús Martín Barbero en 1987, De los medios a las mediaciones, en el cual se hace propone uno de los más importantes constructos teóricos de la comunicación latinoamericana. Según Martín Barbero, no son únicamente los límites del modelo hegemónico lo que han exigido cambiar de paradigma, sino más bien los procesos sociales los que están cambiando el objeto de estudio a los investigadores de comunicación. "Para percibir esto no hay más que ojear los títulos de seminarios y congresos latinoamericanos sobre comunicación en estos últimos cinco años y constatar la presencia obsesiva de los términos transnacionalización, democracia y cultura y movimiento popular" (p. 224).

Ahora bien en este mismo orden de ideas, el eduentretenimiento es una estrategia de comunicación para el cambio social y como tal propone sus mensajes alejados de lo que Martín Barbero denomina en la cita anterior dispositivos meramente tecnológicos, mercantilistas o políticos. De esta forma, la comunicación para el cambio social es usada como una dinámica humana para promover mensajes y valores localmente identificables, sea en

M. Piccini, La imagen del tejedor. Lenguajes y políticas de la comunicación, ps. 40-41 G. Gili, México, 1988. 
una comunidad, en los contenidos de los medios a través de propuestas educativas y de comunicación. Por tanto, dentro de la comunidad en la cual se llevó a cabo la investigación (La Boquilla), se establecieron estrategias de comunicación verbal y no verbal a lo largo de cinco talleres desarrollados como herramienta metodológica, en donde se constituyó un modelo a seguir para la población a educar con entretenimiento, la cual abarcó, como ya se ha explicado, niños y niñas con edades entre 6 y 13 años.

\section{La comunicación verbal y no verbal en la ejecución de talleres educativos}

El concepto de comunicación utilizado fue el propuesto por Mario Kaplún en su libro Hacia nuevas estrategias de comunicación en la educación de adultos, quien la establece como "el proceso por el cual un individuo entra en cooperación mental con otro hasta que ambos alcanzan una conciencia común" (Noseda, p. 11, citado en Kaplún). De la misma forma, Kaplún hace referencia a lo abordado por Antonio Pasquali: “...la relación comunitaria humana que consiste en la emisión/recepción de mensajes, entre interlocutores en estado de total reciprocidad" (Pasquali, p. 51). Determinado este concepto, es posible afirmar que la interacción entre los sujetos que participan en el proceso educativo está apoyada en la comunicación entre los mismos. Por lo tanto la comunicación es el soporte de la educación. La educación y la comunicación requieren, entonces, de un vínculo mutuo, de tal manera que es válido afirmar que la educación necesita de la comunicación para concretarse; asimismo, también es cierto que una comunicación adecuada no podrá lograrse de no mediar un proceso educativo que permita el desarrollo de la capacidad de comunicarse. Esta es una de las principales tesis que demuestra este trabajo. En este orden de ideas, durante la ejecución de los cinco talleres de comunicación se hizo evidente la contraposición al patrón tradicional, vertical y autoritario que concibe a la comunicación como transmisión unilateral de mensajes de un emisor a un receptor, dando lugar a una concepción innovadora y eficaz de comunicación que propone el trabajo con el actor social vinculado con el eduentretenimiento.

\section{La participación como ejemplo de construcción de redes sociales}

Manuel Vidal (1991), expone tres tipos de participación, cada una de ellas presentada en páginas anteriores. La concepción que asume la participación como Comunicar, intervenir y tomar parte fue central para este estudio. Según Vidal, es aquella en la cual hay comunicación e intervención de todos en la solución de un conflicto, durante todas las etapas del mismo, desde su definición hasta su solución. Así pues, se fomentó entre los niños y niñas la participación ciudadana, a través de diversos talleres lúdicos en donde los menores se atrevieron a proponer alternativas ante problemáticas vividas en sus comunidades (La Boquilla). En relación con esto, Nicolás Pineda Pablós asegura que "se trata también que el desarrollo se construya no sólo sobre la base de proyectos de inversión, obras de infraestructura y atracción de empresas extranjeras, sino también y de manera determinante sobre la elevación de la dignidad de las personas involucradas y la valoración de su voz y participación en la construcción de futuros comunes" (Pineda, 1999, 11).

En este orden de ideas, los grupos y las organizaciones son los que asumen y dirigen su propio proceso educativo. El conocimiento es producto de una construcción colectiva, de una búsqueda común en la que todos los educandos aportan, participan, a partir de sus experiencias, de su saber y de una investigación que es también asumida participativamente (Kaplún, 1983, p. 3). De esta manera, la educación misma ya no se ve como un fin en sí, sino como un apoyo imprescindible para la participación. $\mathrm{Su}$ objetivo central es formar y preparar para la participación social. Por tanto, dentro de esta investigación de tipo cualitativa se utilizó el eduentretenimiento como vehículo facilitador para implementar la educación no formal y generar empoderamiento político en niños y niñas de las dos instituciones educativas de las poblaciones mencionadas anteriormente. Relacionado con esto, Mario Kaplún propone una concepción entre emisor y receptores; para utilizar el neologismo acuñado por Cloutier todo hombre es un «emirec», esto es, un emisor/receptor dotado y facultado para ambas funciones. Todos los seres, grupos, comunidades y sectores de la sociedad tienen derecho a participar en el proceso de comunicación actuando, de manera alternada, como emisores y receptores. El modelo comunicacional sustentado podría, pues, ser expresado por dos «emirecs» (ER) intercambiando mensajes.

\section{Aspectos metodológicos del estudio aplicados en La Boquilla}

Una mirada a la comunicación para el cambio social como generadora de participación y empoderamiento político de la población infantil de Cartagena a través de la implementación de estrategias del eduentretenimiento, es un estudio cualitativo. Ahora bien, el trabajo asume por investigación cualitativa aquella que "se interesa más en saber cómo se da la dinámica o cómo ocurre el proceso en que se da el asunto o problema" (Vera, p.1, parra. 2). El diseño cualitativo de esta investigación, se origina en la cotidianidad de los niños y niñas participantes, desde sus necesidades, sus ideales y la de vincularlos al proceso de solución de sus propios problemas. A partir de esto, fue posible crear dinámicas de acercamiento con ellos para así facilitar la ejecución y comprensión de los talleres. En este sentido, "la investigación cualitativa se basa ante todo en el proceso mismo de recolección y análisis. Recordemos que es interpretativa, ya que el investigador hace su propia descripción y valoración de los datos" (Hernández, 2006, p. 6). Por su parte, Fernández, N, en su libro Investigación y técnica de mercado (2008, p. 84), se refiere a la observación cualitativa como un proceso semiestructurado o nada estructurado de captación de información general, sobre la conducta o el comportamiento de las unidades muéstrales, con el fin de realizar un análisis cualitativo de la información resultante. 


\section{Técnica: la etnografía}

Álvarez - Gayou (2003, citado en Hernández Sampieri, 2006 p. 697) considera que el propósito de la investigación etnográfica es describir y analizar lo que las personas de un sitio, estrato o contexto determinado hacen usualmente; así como los significados que le dan a comportamientos realizados bajo circunstancias comunes o especiales y finalmente, presenta los resultados de manera que se resalten las regularidades que implica un proceso cultural. "Los estudios etnográficos investigan grupos o comunidades que comparten una cultura. Ofrecen descripciones detalladas del sitio, los miembros del grupo sus estructuras, procesos, categorías y temas culturales" (Hernández Sampieri, 2006, p. 700). De esta manera, en el caso del estudio concreto, se busca conocer y vincular cada una de las representaciones de la vida cotidiana de los menores, con el fin de obtener resultados que faciliten y favorezcan la investigación. La Boquilla a pesar de estar ubicada cerca de la ciudad de Cartagena de Indias, patrimonio histórico y cultural de la humanidad y de ser un polo turístico en pleno proceso de desarrollo, presenta condiciones de alta vulnerabilidad y de extrema pobreza, debido al deterioro ambiental. Esto conlleva a una disminución de la explotación de sus recursos naturales y a un aumento en las tasas, de desempleo. Por otra parte, esta población, especialmente la infantil, carece de espacios y oportunidades para acceder en sus tiempos libres a programas recreativos y culturales. La recreación debe entenderse como una parte fundamental para el desarrollo integral del niño.

En relación con esta última descripción, cabe resaltar que una de las problemáticas más representativa es la relacionada con la educación tradicional, la cual Kaplún expone como exógena, es decir, concebida desde fuera del sujeto educando, externas a él, o lo que es igual una educación unidireccional en donde el emisor envía un mensaje a un receptor pasivo. Guber en su libro La etnografía, método, campo y reflexividad, la define como "el conjunto de actividades que se suele designar como "trabajo de campo" y cuyo resultado se emplea como evidencia para la descripción. Los fundamentos y características de esa flexibilidad o "apertura", radican precisamente en que los actores y no el investigador, son los privilegiados para expresar en palabras y en prácticas el sentido de su vida, su cotidianeidad, sus hechos extraordinarios y su devenir" (2001, p. 16). Es en este sentido como se concibe el trabajo realizado en el sector de la Boquilla.

\section{Los sujetos escogidos: los niños de la Boquilla y el eduentretenimiento}

La muestra escogida para la realización de esta investigación fue conformada por niños y niñas con edades entre los 6 y 13 años del sector La Boquilla de Cartagena de Indias, pertenecientes al estrato 0 y 1 (bajo), y que cursan 2do, 3ro, 4to y 5 to grado de primaria, identificados por el sistema educativo de su institución como estudiantes con altos índices de indisciplina. Para la selección de estos rangos de edad, se tuvieron en cuenta aspectos de corte psicológico. Según lo expuesto por María Cruz Chacón Blanco, en su libro Desarrollo de la adolescencia y la juventud, la edad entre los 6 y 8 años comprende el periodo de la niñez, caracterizado por ser la etapa más apropiada para la educación a través del juego simbólico, condición propicia para desarrollar el estudio tal como se propuso. A partir de estos argumentos se procedió a seleccionar la muestra. En el corregimiento de La Boquilla la población escogida para esta investigación, la muestra escogida fue de 25 niños y niñas de segundo grado de primaria, pertenecientes al calendario $A$ con edades entre 6 y 8 años del INETEB. La segunda muestra estuvo conformada por 28 niños y niñas de calendario $B$ que cursan tercer grado de primaria de 8 a 12 años de la misma Institución. En este sentido, es relevante citar a Hernández Sampieri y su libro Metodología de la investigación (2006), "la muestra es, en esencia, un subgrupo de la población. Se puede decir que es un subconjunto de elementos que pertenecen a ese conjunto definido en sus características al que llamamos población".

\section{Recolección de datos: Instrumentos}

Se desarrolló un proceso a través de la comunicación verbal (poemas, canciones, conversatorios, explicaciones a partir de sus vivencias) y la no verbal (bailes, dibujos, rally, talleres de relajación para estimular su imaginación, dinámicas en grupo e individuales), para generar en los niños y niñas participantes empoderamiento político y participación en sus procesos de educación. El investigador es quien, mediante diversos métodos o técnicas, recoge los datos (él es quien observa, entrevista, revisa documentos, conduce sesiones, etc.). No sólo analiza, sino que es el medio de obtención de los datos. En la indagación cualitativa, el instrumento no es una prueba estandarizada ni un cuestionario, ni un sistema de medición (Hernández, 2006, p. 583). En este orden de ideas, los instrumentos permiten verificar y registrar la información, utilizando diferentes elementos tales como: grabadoras, cámara de video, formatos, cuestionarios, talleres, folletos entre otros. Sin embargo fueron dos los instrumentos para recoger la información. Por una parte, grupos focales y por otra observación participante.

\section{Grupo focal}

Los grupos focales permiten generar espacios para el intercambio de experiencia y fortalecimiento de los procesos, dando lugar a nuevos espacios de participación y nuevas dinámicas de comunicación, que contribuyan a las relaciones pro-sociales (de comportamiento) de los niños participantes. "Los grupos focales como una estrategia de investigación son apropiados cuando se está interesado en como los individuos forman un esquema o perspectiva de un problema" (Mertens, 2004, p. 245). Con lo anterior, se facilitó la forma en que se llevó a cabo la investigación dado que los niños y niñas interactuaron de manera activa, al proporcionar puntos de vistas diferentes que enriquecieron el proceso de solución de conflictos. 


\section{Observación participativa}

A partir de esta técnica se realizó una mirada al contexto de los niños y niñas participantes en la cual se estudió, analizó y determinó cada una de sus experiencias de vida, las cuales se hicieron evidentes con la ayuda del desarrollo de cinco talleres lúdicos de aprendizaje no formal. "La observación es una técnica que permite obtener información mediante el registro de las características o comportamientos de un colectivo de individuos, o elementos sin establecer en un proceso de comunicación y por tanto sin la necesidad de colaboración por parte del colectivo analizado" (Fernández, 2008, p. 84). A través de la observación participativa fue posible interactuar y ser parte de la cotidianidad de los niños y niñas, para así adentrarnos en la situación o problema a tratar, brindándoles nuevas dinámicas de comunicación que contribuyan en sus relaciones pro- sociales. Por tanto, a través del eduentretenimiento en contraposición a la educación formal se desarrollaron dinámicas y juegos que generaron un ambiente de armonía y confianza con los niños y niñas participantes. Con esto, se puede plantear uno de las principales ideas de este artículo:

La búsqueda de una educación que genere oportunidades para todos es una lucha a nivel mundial. El objetivo es integrar a todas las personas en un mismo entorno. El ser humano no se debe adaptar al sistema educativo; al contrario, el sistema debe ser adaptado para él, pues la educación de personas debe ser parte integral de la planificación de la enseñanza, la elaboración de planes de estudio y la organización escolar en general (Sandoval, 2011, p. 166).

Ahora bien, todo el proceso se caracterizó por ser un proceso lento y arduo pero que resultó productivo para los menores, puesto que sus niveles de autoconfianza resultaron favorecidos al reconocerse como personas capaces de trasformar las problemáticas y vivencias con su entorno tanto familiar como educativo. Al mismo tiempo fue vital para desarrollar procesos de auto-reconocimiento como seres capaces de construir su propia realidad teniendo en cuenta al otro como sujeto. En este sentido, la técnica de observación participante fue útil dado que a través de ella es posible la descripción de grupos sociales y escenas culturales mediante la vivencia de las experiencias de las personas implicadas en un grupo o institución, con el fin de captar cómo definen su propia realidad y los constructos que organizan su mundo. Jorgensen en su libro La observación participativa: Metodología para el estudio humano se refiere a ésta como generadora de prácticas y verdades teóricas sobre la vida humana, basadas en la realidad de la existencia diaria, la cual tiene un especial interés en el pensamiento humano y la interacción, vista desde la perspectiva de quienes están involucrados o sus situaciones particulares (1989, pp. 13-14).

\section{Descripción de actividades}

De acuerdo con lo planificado, se llevaron a cabo cinco talleres lúdicos de comunicación, los cuales se ejecutaron en un grupo focal conformado por niños y niñas entre 6 y 13 años del sector de La Boquilla de la ciudad de Cartagena. Las actividades desarrolladas incluyeron rallys, conversatorios y dinámicas en grupo e individuales, todas con el fin de generar en los actores sociales procesos de participación y empoderamiento político. A continuación una breve descripción de las cinco actividades de campo desarrolladas.

\section{Actividad \#1. Participación: cuando juego, cuando opino, cuando me integro... estoy participando!}

Objetivos: Crear un ambiente de distensión, armonía y confianza propicias para la participación colectiva de los niños.

Participantes: Esta actividad se enfocó para grupos de niños y niñas entre 6 y 13 años del corregimiento de La Boquilla.

Tiempo: La charla duró 15 minutos aproximadamente. Cada juego tuvo una duración de 20 a 25 minutos máximo (40 minutos). Para las reflexiones se destinaron 20 minutos adicionales.

Materiales: Video beam, diapositivas, marcadores, papel periódico, hojas de block y cámara fotográfica.

\section{- Procedimiento}

Después de las primeras visitas realizadas al el corregimiento de La Boquilla para observar el comportamiento de los niños y niñas y generar reconocimiento con los mismos, se puso en marcha la primera actividad basada en una charla acerca de la participación. De manera relacionada, se propuso el juego “Teléfono Roto”, en donde la finalidad consistió en descubrir las barreras e interferencias de la comunicación entre los niños. Se dividió el grupo en dos equipos, nombrando al niño o niña que encabezaría la fila. A cada equipo se le mencionó un mismo mensaje, el cual fue transmitido de niño en niño de manera secreta. Luego se preguntó al último niño o niña de cada fila cuál era el mensaje que se comunicó. Posteriormente se confrontó con el mensaje real transmitido por primera vez, y al final se analizó el primer mensaje y el último confrontando las diferencias. Las frases utilizadas fueron las siguientes:

- ¡Qué triste está Tristán, por tan tétrica trama teatral!

- Para la Lola una lila que le di a la Adela.

- Si vas a la cocina y ves un melón... acuérdate de tu amigo que te quiere un montón.

- Paco Peco, chico rico, le gritaba como loco a su tío Federico.

- Tres traperos tapan con trapos la tripa del potro.

- El perro de San Roque no tiene rabo, porque Ramón Ramírez se lo ha robado.

- El Toro y el mono en la torre del oro cantan en coro.

- Tres tristes tigres, trigraron en un trigal tres pedazos de trigo.

- ¿Cómo quieres que te quiera?, si quien quiero que me quiera no me quiere. 
- Contigo entró un tren con trigo, pero el tren que entró con trigo se estrelló.

De la misma forma, se dio lugar al segundo juego "Representaciones". En primer lugar, se dividió el grupo en dos bloques, se eligió un representante por subgrupo. Cada representante debía extraer un papel que podía tener el nombre de un animal, un estado de ánimo, una acción o un objeto. Una vez el niño representante seleccionara el papel, lo dibujaba a su vez en un papel periódico, con el fin que el resto de su grupo adivinara lo que había dibujado en el menor tiempo posible. El grupo con mayor número de aciertos sería el ganador. Las palabras que se encontraban en los papeles relacionadas con animales, estados de ánimo, acciones y objetos fueron las siguientes:

Animales: gato- perro- pato- sapo- paloma- culebra- león- pezoso- mariposa-oveja- cerdo- curro- pollito- ratón.

Estados de ánimo: felicidad- tristeza- odio- llanto- susto- sueño- hambre.

Acciones: correr- nadar- jugar- pelear- tomar.

Objetos: carro-casa- puerta- lámpara-botella- libro-lápiz- motomartillo- mesa-computador-celular-balón- dulces- gorra- pantalón-zapatos. Al finalizar las dinámicas, se realizaron reflexiones en donde los niños y niñas identificaron los aspectos positivos y negativos en los aprendizajes del día y se reforzó el concepto de participación que se les explicó al iniciar el taller.

\section{Actividad \#2. Democracia: ¿Cuáles son mis derechos y deberes como niño o niña?}

Objetivos: Fomentar la afinidad y confianza entre los niños y niñas, a través de la elección de un representante por grupo (amarillos y verdes), al tener un contacto directo con el líder a quien han elegido.

Diseñar un dibujo realizado por cada niño y niña, que contenga la explicación de lo entendido durante el taller de democracia.

Participantes: Dividir el grupo en dos subgrupos al azar; el criterio de selección lo daba el color extraído de una bolsa por cada niño y niña (amarillos y verdes).

\section{Tiempo}

Para la selección al azar de los 2 subgrupos se destinaron 10 minutos.

En cuanto a la postulación y sufragio de un representante por subgrupos, se estimó un tiempo aproximado de 10 a 15 minutos. Para la realización del diseño de los dibujos acerca de lo que cada niño entendió por democracia se estableció una duración de 30 a 45 minutos.

Al finalizar la actividad, se reforzó el concepto aprendido, en este caso democracia, con el fin que cada uno de los participantes conozca o refuerce sus conocimientos acerca de los derechos y deberes que tienen en su condición de ser niños o niñas.

Materiales: Video beam, diapositivas con la información necesaria, papeles de colores (Amarillo, Azul y Rojo), hoja de block, colores, marcadores delgados y cámara fotográfica.

\section{- Procedimiento}

Esta actividad propuso poner en práctica el concepto de democracia. En primera instancia, se dictó una charla acerca de lo que es el concepto de "democracia" adaptada a contextos cotidianos de los niños y niñas participantes, tomando como primer ejemplo a la familia hasta llegar a las votaciones estudiantiles. Las actividades que se implementaron fueron las siguientes: dividir a los niños y niñas en dos subgrupos escogidos al azar, de manera que los niños y niñas seleccionarán un papel (amarillo, azul o rojo); de esta manera quedaron establecidos tres subgrupos. Una vez se reunieron los tres subgrupos, se dio lugar a la elección de uno de los niños y niñas, postulados como líderes de cada grupo (amarillo, azul o rojo). Así pues, elegidos los tres líderes que representaron a cada subgrupo, se encargaron de repartir hojas y colores a cada uno de los miembros de su grupo, con el fin de dibujar lo que cada uno aprendió durante la charla del concepto de "democracia". El rol de los líderes de cada grupo no solo fue la de repartir las hojas y los colores, sino también la de velar por el cuidado y adecuado uso de los implementos, es decir, que los niños compartieran los colores y fueran responsables de regresarlos en buen estado.

\section{Actividad \#3. Reconocimiento del otro: ¿Quién es el otro y por qué es importante reconocerlo?}

Objetivo: Explicar, a través de técnicas asociadas al eduentretenimiento, a los niños y niñas participantes del taller en qué consiste reconocer al otro.

\section{Participantes: Los cuatro subgrupos.}

Tiempo: La charla tuvo una duración de 10 minutos. El diseño de las carteleras duró de 30 a 45 minutos. La explicación y exposición de las carteleras por grupo duró un tiempo de 5 a 10 minutos.

Materiales: Video beam, diapositivas, pliegos de papel periódico, revistas, goma, tijeras, hojas de block y colores, cámara fotográfica.

\section{Procedimiento:}

Para la realización de esta actividad, se hizo necesaria una charla acerca de la importancia que tiene el reconocer al otro como sujeto, sin importar las diferencias que existen entre las personas. Se hizo énfasis en la siguiente tesis: "desde las diferencias es posible construir ciudadanía, y que el ser humano se legitima en la medida en que reconoce al otro". Después de la charla se cerró el taller con una actividad artística, en donde los niños y niñas se organizaron en cuatro grupos seleccionados al azar, con el fin establecer diversos grupos de niños y niñas. De esta manera se 
puso en práctica la temática del taller, es decir "reconocimiento del otro". La actividad consistía en la construcción colectiva de una cartelera por subgrupos, en donde los niños y niñas podían dibujar, recortar, pegar y escribir lo que representaba para ellos el tema de "reconocer al otro". Para finalizar, cada grupo socializó a los demás compañeros la cartelera diseñada, expresando lo aprendido en el transcurso de la jornada.

\section{Actividad \#4. Retroalimentación: analicemos lo que hemos aprendido}

Objetivos: Desarrollar el proceso de retroalimentación por parte de los niños sobre cada uno de los conceptos aprendidos.

Participantes: Todos los niños y niñas asistentes a la actividad.

Tiempo:- La mesa redonda duró de 20 a 30 minutos. La proyección de la película tuvo una duración de 1 hora y 24 minutos. Las reflexiones se realizaron en 20 minutos.

Materiales: Video beam, computador portátil con parlantes, DVD de la película "vecinos invasores", cámara fotográfica.

\section{Procedimiento:}

Para la realización de esta actividad se hizo, en primera medida, una mesa redonda con los niños y niñas asistentes la cual tuvo la finalidad de aclarar las dudas que manifestaban los menores acerca de los tres temas tratados en las actividades pasadas ("participación", "democracia, "reconocimiento de otro"). Después de la sección de preguntas y respuestas, en donde quedaron claros los conceptos tratados con anterioridad, se dio paso a la proyección de la película "Monsters Inc.", cuya trama se basa en la mayor empresa de miedo del mundo. Al finalizar la proyección del film, cada niño y niña realizó reflexiones sobre los temas estudiados durante los talleres en donde ejemplificaron con hechos y vivencias de su cotidianidad lo aprendido en el taller, y que luego fueron comparadas con lo visto en la película.

\section{Actividad \#5 Clausura: comunicación verbal y no verbal.}

Objetivos; Realizar un conversatorio con los niños y niñas del corregimiento de La Boquilla de la ciudad de Cartagena, con relación a las temáticas abordadas en los talleres pasados.

Participantes: Todos los niños y niñas asistentes a la actividad.

Tiempo: El conversatorio tuvo una duración de 30 minutos. La entrega y lectura de los folletos que contenían un resumen de lo visto en los talleres anteriores fue de 20 minutos. Para la estimulación de los menores con canciones de relajación se estimaron 30 minutos. Para la representación de lo aprendido a través de la comunicación verbal (poemas, canciones) o no verbal (dramatizados, bailes, dibujos, etc.) se destinaron 40 minutos.
Materiales: Computador portátil, parlantes, canciones de relajación del pianista Jannin, folletos, cámara fotográfica.

\section{Procedimiento:}

Para la realización de la quinta actividad, con la que se clausuraron las actividades bajo el marco del taller: "el eduentretenimiento como vehículo facilitador para generar procesos de participación y empoderamiento político", se llevaron a cabo los siguientes pasos: en primera instancia, se desarrolló un conversatorio con los niños y niñas en el cual se abordaron cada uno de los temas de las actividades realizadas anteriormente. Asimismo, se efectuó la entrega de folletos que contenían un resumen de las temáticas tratadas en los talleres. De igual manera, se estimuló la imaginación y la relajación de los menores con la ayuda de canciones de relajación en donde sus sentidos: vista, oído y tacto entraron en contacto al reconocerse como seres capaces de contribuir con un proceso personal que está en construcción. Para la finalización de esta quinta y ultima actividad, se dio paso a que los niños y niñas dejaran volar su imaginación, siendo libres de escoger una forma diferente de expresar lo que aprendieron y entendieron durante este proceso, contando con la ayuda de la comunicación verbal (poemas, canciones) o no verbal (dramatizados, bailes, dibujos, etc.). Así pues, los menores se convirtieron en los gestores de la conclusión de las actividades de manera dinámica, diferente, lúdica e innovadora.

\section{Resultados y recomendaciones descriptivas para imple- mentar estrategias de eduentretenimiento en la Boquilla, como forma de transformar la educación tradicional en educación para el cambio social.}

En este último apartado se describirán de manera breve los resultados obtenidos a través de los talleres ejecutados en La Boquilla, los cuales apuntaron a resolver la pregunta central planteada desde el inicio de este documento. Para tal fin, se hizo pertinente la utilización del eduentretenimiento como herramienta comunicacional para abordar la educación no formal, dado que a lo largo del desarrollo de las actividades ejecutadas en dichas comunidades las respuestas ante este nuevo modelo pedagógico fueron puestas en práctica por los niños y niñas participantes.

\section{Actividad \# 1 Participación}

A través de este taller, los niños asumen como propio el concepto de participación que propone dicha categoría como la única manera civilizada de resolver los conflictos y las diferencias entre los seres humanos. A través del taller, los niños y niñas aprenden la importancia que tiene la participación con relación a la cooperación dentro de un grupo o comunidad, debido a que cada uno de los juegos desarrollados se convirtió en la manera de ganar puntos por grupos, en donde el grupo con mayor número de puntos sería el ganador. Algunas de las reflexiones de los niños y niñas apuntaban en este sentido: "lo malo fue 
que algunos compañeros no querían jugar porque tenían pena y eso nos hizo perder puntos" (Samir Díaz, 8 años, institución INETEB)... "Gracias a la participación en equipo pudimos ganarle a los demás grupos, porque si nos da pena decir lo que pensamos nunca hubiéramos ganado" (José Domingo Zabaleta, 9 años, de la institución educativa técnica de la Boquilla).

\section{- Actividad \# 2 Democracia}

El concepto de democracia fue relacionado con la vida cotidiana de los niños y niñas, es decir, para entenderlo fue necesario acudir a sus entornos cercanos tales como la familia y la misma escuela (votaciones estudiantiles). Una vez asumido el concepto como forma de participación respetuosa de los intereses, ideas y principios de los demás, es decir, una vez asociada la democracia con procesos cuya expresión y concreción es la organización popular de base, en cuanto ésta se propone ganar para sus integrantes y para el conjunto de los sectores populares un espacio social, los menores empezaron a mostrar un total interés a la temática abordada, puesto que expresaban su punto vista sin ningún temor, motivados por lo relacionado con los derechos que tienen por ser niños y niñas. Todo este contexto generó que manifestaran experiencias vividas que fueron puestas como ejemplo personal a toda la comunidad. Mediante la dinámica de elección del representante grupal a través de votaciones quedó claro para los menores que los ideales y pensamientos que posee cada persona deben ser respetados por los demás, dado que al momento de las votaciones cada niño y niña tuvo la autonomía de elegir quien le parecía mejor como representante del grupo.

\section{- Actividad \# 3: Reconocimiento del otro}

Con este taller, se logró incentivar la autoconfianza de los participantes de las actividades, así como también la seguridad y asertividad, dándole importancia a la toma de decisiones colectivas, enfocando a cada niño y niña a reconocer al otro como persona y así organizarse para alcanzar un bien común. Atrás quedó la educación tradicional; la propuesta apuntó a educar con entretenimiento, confirmándose la idea de partida de este estudio relacionada con la ausencia de procesos educativos que estimule y prepare para la participación, en donde el eduentretenimiento puede ser propuesto como una estrategia eficaz de enseñanza en la medida que brinde herramientas novedosas a los receptores. Por otra parte, la comunicación para el cambio social es un proceso largo y lento, no se da espontáneamente ni de un día para otro, por lo tanto se requiere de un verdadero compromiso por parte de los participantes (receptores) y de los mediadores o facilitadores (emisores).

\section{- Actividad \# 4 Retroalimentación}

En primer lugar, se efectuó una mesa redonda con los niños y niñas asistentes, dando lugar a una sección de preguntas y respuestas con relación a los tres temas abordados durante las actividades pasadas (temas: "participación”, "democracia”, "reconocimiento del otro"). Una vez los niños y niñas tuvieron claros estos tres conceptos, se les proyectó la película "Monsters Inc." la cual muestra la importancia que tiene reconocer a los demás sin importar las diferencias que puedan existir entre los sujetos. Así mismo, el trabajo en equipo propuesta por la película fue relacionado por los niños y niñas con el taller de participación. Luego de dicha proyección, los niños y niñas hicieron reflexiones articuladoras de los tres conceptos abordados a través de las actividades. En este contexto fueron importantes las vivencias mostradas por los personajes de la película "Monsters Inc.". A partir del audiovisual, los niños y niñas decidieron poner ejemplos de su vida cotidiana, dando lugar a una contextualización real de lo aprendido a lo largo de los anteriores talleres.

\section{- Actividad \# 5 Clausura}

El trabajo con los niños y niñas de La Boquilla deja como conclusión la posibilidad de implementar a través del eduentretenimiento, y técnicas de comunicación no masificadas modelos participativos. Dichos modelos ofrecen a las estructuras sociales sistemas de interacción contrarios a la unidireccionalidad y verticalidad que imperan en la comunicación transmisora tradicional. Esto se puede sustentar con el siguiente extracto:

...nos enfrentamos a la necesidad de promover, facilitar y animar el fortalecimiento de la capacidad expresiva, comunicativa y lúdica de sus habitantes, los lazos, los intercambios, las redes sociales cotidianas y los lazos entre las organizaciones y los movimientos sociales, que son la base para el fortalecimiento de la esfera pública y del tejido social en su conjunto (Pereira, Bonilla, et al, 1998, citado en Beltrán, 2002, p. 87).

En este sentido, de una u otra manera se rompen los paradigmas de las lógicas del consumo; los grupos y las comunidades pasan a intervenir directamente en el proceso comunicacional y a emitir mensajes. Si en la comunicación educativa grupal los destinatarios recepcionan un mensaje producido por otros sobre el que luego reflexionan, la participativa da un paso más: los grupos se convierten en emisores, en productores de mensajes.

\section{Conclusiones}

De manera puntual, y a partir del estudio desarrollado con los niños del sector de La Boquilla de Cartagena, se pueden extraer las siguientes conclusiones:

- El eduentretenimiento es una técnica innovadora que contribuye a que los sujetos aprendan a reconocerse como diferentes, plurales y con voz propia.

- Lo anterior trae como consecuencia la posibilidad de transformar el tejido social, dado que las formas de relacionarse de los sujetos varían, y a su vez, de fortalecer los procesos de cohesión social al interior de las comunidades.

- Con los talleres desarrollados en la Boquilla, es posible concluir que en la medida que los actores sociales se 
apropien de su narración, de sus formas de contar, de sus formas de expresar, se construirá de manera simultánea una plataforma para que los contenidos desarrollados ayuden a que la gente cree sus propios sentidos, los reconozca, los fortalezca y desde aquí transforme su entorno.

- Los contenidos en este estudio trabajados han aportado en el proceso para que los niños de la Boquilla se transformen en ciudadanos. Desde este punto de vista, la comunicación es catalizadora de procesos de apropiación simbólica, es decir, de re-codificación del propio ser, lo que implica el fortalecimiento de las identidades locales influyentes en los proyectos de vida futuros de la misma comunidad.

\section{Referencias}

Ardila, E. (2010). Concepciones de los docentes de Ciencias Sociales sobre la naturaleza del pensamiento crítico. Revista Aletheia, Revista de desarrollo humano, educativo y social contemporáneo. [Revista electrónica], Vol. 2. Número 1. Disponible en: http://aletheia.cinde.org.co/index.php/ALETHEIA/article/ view/15/12

Batliwala, S. (1993). Empowerment of Women in South Asia: Conceptsand Practices (second draft). Asian-South Pacific Bureau of Adult Education.

Beltrán, L. (2002). La comunicación y el desarrollo democrático en América Latina. Cadiz: Universidad de Cadiz-España. Extraído el 19 de julio de 2011 de http://gumilla.org/biblioteca/bases/ biblo/texto/COM2004126_79-95.pdf

Bordenave, J. (1976). Communication of agricultural innovations in latin America. The need for news models. Communication Research, Vol 3, No 2

Chacón, M. (2009), Desarrollo de la adolescencia y la juventud. Revista Innovación y experiencias educativas, 25, 1-16. Disponible en: http://www.csi-csif.es/andalucia/modules/mod_ense/ revista/pdf/Numero_25/M_CRUZ_CHACON_1.pdf

Chaparro, M. (2009) Comunicación para el empoderamiento y comunicación ecosocial, la necesaria creación de nuevos imaginarios. Perspectivas de la comunicación, 12, 1. 146-158. Disponible en: http://www.perspectivasdelacomunicacion. cl/revista_1_2009/parte3_03.pdf

Fernández, N. (2008). Investigación y técnica de mercado. Madrid: ESCIC.

Freire, P. (2007). La educación como práctica de la libertad. Madrid: Siglo XXI. Disponible en: http://books.google.com/books?i $\mathrm{d}=\mathrm{tGCD} 2 \mathrm{i} 9 \mathrm{zZ58C}$ \&printsec $=$ frontcover\&hl $=$ es\&source $=\mathrm{g}$ bs_ge_summary_r\&cad=0\#v=onepage\&q\&f=false
Freire, P. (2004). Cartas a quien pretende enseñar. Buenos Aires: Siglo XXI.

Freire, P. (2003), Lecciones de Paulo Freire, cruzando fronteras: experiencias que se completan

Freire, P, (1970) Pedagogía del oprimido. Rio de Janeiro: Editor paz y tierra. m, R. (2001). La etnografía, método, campo y reflexividad. Bogotá: Editorial Norma.

Gumucio, A. (2001b). Haciendo Olas, Historias de comunicación participativa para el cambio social. La paz: Fundación Rockefeller.

Gumucio, A. \& Tufte, T. (2008). Antología de la comunicación para el cambio social. La paz: Plural editores.

Jorgensen, D. L. (1989). La observación participativa: Metodología para el estudio humano. Newbuey, CA: sage

Kaplún, M, (1983). Hacia nuevas estrategias de comunicación en la educación de adultos. Santiago de Chile. OREALC

Kaplún, M. (2002). Elcomunicadorpopular. La Habana: Caminos. Disponibleen:http://www.captel.com.ar/downloads/2906061524_ kaplun_el\%20comunicador\%20popular.pdf

Kaplún, M. (1978a). Educar ¿Para qué? Quito: CIESPAL.

Kaplún, M. (1978b). Cassete-foro: un sistema de comunicación participatoria. Quito: CIESPAL.

Hernández, R, (2006) Metodología de la investigación. México: McGrawHill.

Marques de Melo, J. (1979). Pensamiento comunicacional brasilero. Sao Bernardo do Campo, Umesp.

Martín Babero, J. (1999). Las transformaciones del mapa: identidades, industrias y culturas, en Garretón, M. A. (Comp.) América Latina: un espacio cultural en un mundo globalizado (Bogotá: Convenio Andrés Bello) 296-331.

Martín-Barbero, J. (1987). De los medios a las mediaciones: comunicación, cultura y hegemonía. México: Gustavo Gilli.

Martín Barbero, J, (2002b) La educación desde la comunicación, Editorial Norma, III. Reconfiguraciones comunicativas del saber y del narrar.

Ministerio de educación, (1994) Capitulo 2 de la Ley 115, Programas técnicos ofrecidos por instituciones de Educación no formal, Disponible en: http://www.mineducacion.gov. co/1621/article-87076.html. 
Moreno, M. (2002). El pensamiento del profesor: evolución y estado actual de las investigaciones, en Perafrán, G. A. y Adúriz-Bravo, A. (eds.). Pensamiento y conocimiento de los profesores. Debate y perspectivas internacionales (pp. 61-78). Bogotá: Universidad Pedagógica Nacional.

Navarro, L. (2010a). Entre esferas públicas y ciudadanías: las teorías de Arendt, Habermas y Mouffe aplicadas a la comunicación para el cambio social. Barranquilla: Ediciones Uninorte.

Navarro, L. (2010b) Una reflexión sobre los medios de comunicación ciudadanos como esferas públicas, movilizadores de identidades y contraidentidades de los sujetos políticos en Colombia. Revista Encuentros, 15, 33-44.

Navarro, L. (2008). Aproximación a la comunicación social desde el paradigma crítico: Una mirada a la comunicación afirmadora de la diferencia. Revista investigación y desarrollo, 16. social.

Pereira, J., Bonilla, J. et al (1998). La comunicación en contextos de desarrollo, balances y perspectivas. Revista Signo y pensamiento, 32, pp. 119-138.

Restrepo, S. (1998). La evolución de la comunicación para el desarrollo. Londres: Tauris.
Sandoval, R. (2011). Las mediaciones tecnológicas en el campo educativo. Revista Educación y humanismo, vol 13, 21, pp. 162-173.

Sen, G. (1995) El empoderamiento como un enfoque a la pobreza. en I. Arriagada y C. Torres (eds.) Género y pobreza. Nuevas dimensiones, serie Ediciones de las Mujeres $N^{o} 26$, (Santiago, Isis Internacional), julio.

Tufte, T. (2008, junio) El eduentretenimiento: buscando estrategias comunicacionales contra la violencia y los conflictos. [Versión electrónica]. Revista Brasileira de Ciencias de Comunicacao.

Tufte, T, (2005) El eduentretenimiento en Barranquilla: Universidad del Norte. Disponible electrónicamente: http://ciruelo. uninorte.edu.co/pdf/invest_desarrollo/16-2/9_Aproximacion\%20a\%20la\%20comunicacion.pdf

Pineda, (1999), Tres conceptos de ciudadanía para el desarrollo de México. Este País, 101.

Vera, L. La investigación cualitativa. UIPR, Ponce, P.R.

Vidal, M. (1991). Participación, comunicación y organización. Revista Signo y Pensamiento, 19, 22-32. 